\title{
Erratum: Torque undergone by assemblies of single-domain magnetic nanoparticles submitted to a rotating magnetic field [Phys. Rev. B 94, 184420 (2016)]
}

\author{
J. Carrey and N. Hallali
}

(Received 19 July 2018; published 19 October 2018)

DOI: 10.1103/PhysRevB.98.139902

Magnetic interactions were not correctly implemented in the program used to obtain the results shown in Sec. V of the original paper. Figures calculated with the corrected version of the program are provided as well as new comments. A Ph.D. student, acknowledged in this Erratum, has discovered a mistake in the kinetic Monte Carlo simulations that we have used to calculate the curves presented in the article associated with this Erratum. The error concerns the expression used for magnetic interactions: The equation used in the article is correct, but its typing into the program was not correct. We are sorry about that and present our apologies to both the editor and our readers. The former program will be called "program 1 ," and the corrected one will be called "program 2."

The typing error in program 1 modified the orientation of the magnetic interaction vector, modifying the figures in Sec. V (influence of magnetic interactions). The results in the previous sections were not modified. Below, the modified versions of the figures are shown, and new comments for the figures are provided. The text below has been written to be self-consistent and to replace completely Secs. V and VI of the main article.

\section{INFLUENCE OF MAGNETIC INTERACTIONS ON THE TORQUE PROPERTIES}

The influence of magnetic interactions on the torque undergone by an assembly of magnetic nanoparticles (MNPs) is now presented. To avoid an unnecessarily long presentation, the focus is directly put on the experimentally relevant case of an assembly with randomly oriented anisotropy axes. The system under study is a sphere containing $N=10000$ particles; the volume concentration of the particles inside the sphere is $c$. Technically, to generate the position of the MNPs inside the sphere, they are first placed on a cubic lattice and then displaced by a random value in a random direction. The maximum value of the random displacement is chosen so the MNPs cannot overlap with their neighbors. As a consequence, the system under study is a disordered assembly of MNPs. In a typical simulation, the torque as a function of $\phi$ is calculated and the maximum value of $|\tau|$ extracted.

\section{A. Interacting ferromagnetic particles}

In Fig. 13, the properties of ferromagnetic NPs $(T=0 \mathrm{~K})$ are shown. Figure 13(a) illustrates the case of MNPs without anisotropy, and Fig. 13(b) illustrates the one with finite anisotropy values. In the absence of magnetic interactions and for a null anisotropy, the torque is null but increases with magnetic interactions, displays a maximum, and then decreases [see Fig. 13(a)]. Quantitatively, by varying $M_{S}, \mu_{0} H_{\max }$, and $d$, it has been found that the value at which the torque is maximal depends on the $\frac{c M_{S}}{H_{\max }}$ ratio only. The maximum occurs when $\frac{c M_{S}}{H_{\max }} \approx 4$ as shown in Fig. 13(a). In program 1, the maximum occurred for a value of 1 . This shows a main difference between program 1 and program 2: an overestimation of the magnetic interactions in program 1. As a consequence, in all the figures, any peak occurring in a graph where data are plotted as a function of concentration is shifted toward higher concentrations in program 2 compared to program 1 .

The presence of the peak in Fig. 13(a) can be understood qualitatively this way: (i) One first well-known effect of magnetic interactions is to increase the effective anisotropy of MNPs [1], which should, in turn, increase the total torque of the assembly. (ii) A second one is to decrease the total magnetization of the assembly because the MNP magnetizations tend to adopt flux-closure configurations. We have checked this second effect by analyzing data shown in Fig. 13(a): Indeed, the magnetization of the assembly decreases continuously with $c$ (not shown). (iii) The combination of these two phenomena with opposite effects leads to the bell-shaped curves displayed in Fig. 13(a).

Figure 13(b) illustrates the more relevant case of nanoparticles with finite anisotropies. It is recalled that, for magnetically independent MNPs, the torque strongly increases when $\frac{\mu_{0} H_{\max } M_{S}}{2}<K_{\text {eff }}<\mu_{0} H_{\max } M_{S}$ and is maximized for $K_{\text {eff }}$ just below $\mu_{0} H_{\max } M_{S}$ [see Fig. 9(b) of the original paper]. With the parameters used to calculate Fig. 13(b), $\mu_{0} H_{\max } M_{S}=$ $5 \mathrm{~kJ} / \mathrm{m}^{3}$. As a consequence, in the absence of interaction, MNPs displaying the largest torque are the ones with $K_{\text {eff }}$ just below $5 \mathrm{~kJ} / \mathrm{m}^{3}$, and particles with an anisotropy above $2.5 \mathrm{~kJ} / \mathrm{m}^{3}$ display a normalized torque amplitude $\frac{|\tau|}{\tau_{\max }}$ larger than $\frac{1}{\sqrt{N}}$ [see Fig. 13(b)]. The influence of magnetic interactions is quite interesting. For particles which have anisotropies below $2.5 \mathrm{~kJ} / \mathrm{m}^{3}$ and which generate a normalized torque far smaller than $\frac{1}{\sqrt{N}}$ in the absence of magnetic interactions, torque amplitude increases above this value for concentrations higher than $3 \%$ [see the data for $K_{\text {eff }}=1$ to $2 \mathrm{~kJ} / \mathrm{m}^{3}$ in Fig. 13(b)]. This means that they probably enter into a timereversal symmetry-breaking regime. For $K_{\text {eff }}=3$ to $4 \mathrm{~kJ} / \mathrm{m}^{3}$, which is a case where magnetically independent nanoparticles already display a normalized torque amplitude above $\frac{1}{\sqrt{N}}$, magnetic interactions increase the torque further in a given range of concentrations. NPs with $K_{\text {eff }}=5 \mathrm{~kJ} / \mathrm{m}^{3}$ have a 


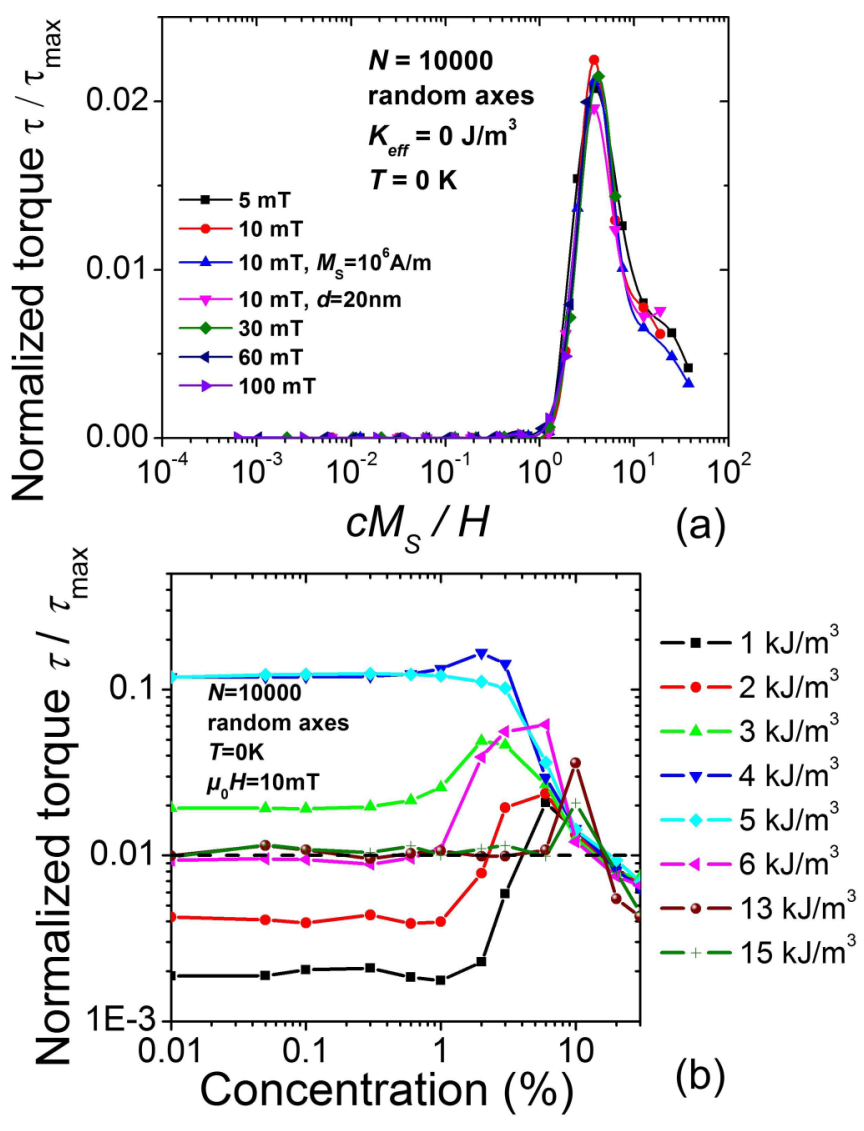

FIG. 13. Influence of the concentration on the torque undergone by an assembly of $N=10000$ interacting ferromagnetic particles with randomly oriented axes at $T=0 \mathrm{~K}$. Unless otherwise specified in the legend, $d=10 \mathrm{~nm}, \mu_{0} H_{\max }=10 \mathrm{mT}$, and $M_{S}=$ $0.5 \times 10^{6} \mathrm{~A} / \mathrm{m}$. (a) $K_{\text {eff }}=0 \mathrm{~J} / \mathrm{m}^{3}$. Normalized torque as a function of $\frac{c M_{S}}{H_{\max }}$ for various values of $\mu_{0} H_{\max }, M_{S}$, and $d$ as specified in the graph. (b) Normalized torque for $K_{\text {eff }}$ varying between 1 and $15 \mathrm{~kJ} / \mathrm{m}^{3}$. The horizontal dashed line represents the function $\frac{|\tau|}{\tau_{\max }}=\frac{1}{\sqrt{N}}$.

normalized torque equal to $\frac{1}{\sqrt{N}}$ in the absence of interactions and for extremely small concentrations $(c \approx 0.0001 \%$, not shown in Fig. 13(b)], but a very small amount of magnetic interactions is enough to increase dramatically the torque amplitude. This explains that, in the range of concentration shown in Fig. 13(b), their torque is very large. Finally, interestingly, NPs with anisotropies above $5 \mathrm{~kJ} / \mathrm{m}^{3}$ which, in the absence of magnetic interactions, display a normalized torque amplitude equal to $\frac{1}{\sqrt{N}}$ generate in a rather narrow range of interactions a torque amplitude increase above this limit. This is probably due to the fact that magnetic interactions can also reduce the effective anisotropy of some particles which, in this case, display a large torque amplitude. More work should be performed in order to check the anisotropy distribution into the assembly so as to check the validity of this hypothesis. For all the studied anisotropy values, torque decreases for the largest values of $c$, which can be explained with arguments similar to the ones cited above for the null anisotropy regime: A large amount of magnetic interactions drops the magnetization of the assembly and thus decreases the torque.

\section{B. Properties at $300 \mathrm{~K}$ for finite diameters}

The behavior of MNPs at $300 \mathrm{~K}$ is presented in Fig. 14 . In Fig. 14(a), the effect of anisotropy is shown for 10-nm NPs submitted to a magnetic field of $\mu_{0} H_{\max }=10 \mathrm{mT}$. In Fig. 14(b), the torque amplitude at $T=0 \mathrm{~K}$ and $T=300 \mathrm{~K}$ are compared for a few selected anisotropy values. NPs with a diameter of $10 \mathrm{~nm}$ begin to present a ferromagnetic behavior for an anisotropy above $5 \mathrm{~kJ} / \mathrm{m}^{3}$. Below this value, they are clearly superparamagnetic. This anisotropy value is above the anisotropy range into which time-reversal breaking occurs for noninteracting NPs $\left(2.5-5 \mathrm{~kJ} / \mathrm{m}^{3}\right)$. As a consequence, noninteracting 10-nm NPs never generate a normalized torque $\frac{|\tau|}{\tau_{\max }}$ above $\frac{1}{\sqrt{N}}$ at $300 \mathrm{~K}$, whatever their anisotropy [see Figs. 14(a) and 14(b)]. Here again, magnetic interactions drive the NP assembly into a regime where the normalized torque exceeds $\frac{1}{\sqrt{N}}$. With the parameters used here, the maximum torque is reached for nanoparticles with $K_{\text {eff }}=3 \mathrm{~kJ} / \mathrm{m}^{3}$ at a volume concentration of $5 \%$. The influence of the diameter is shown in Fig. 14(c) for MNPs with $K_{\text {eff }}=13 \mathrm{~kJ} / \mathrm{m}^{3}$. In this case, the largest torque is obtained for 25-nm MNPs concentrated at 5\%. Finally, Fig. 14(d) shows the influence of the assembly size in a typical case where particles see their torque strongly increased by the presence of magnetic interactions. In the ordinates of this graph, $\frac{|\tau| \sqrt{N}}{\tau_{\max }}$ is plotted, so the direct effect of $N$ on the torque per particle is canceled (see Fig. 11 of the original paper). It permits illustrating the consequences of the interaction strength attenuation only. As expected, both the positive and the negative influences of magnetic interactions on the torque amplitude are strongly reduced when reducing the size of the assembly.

The graphs shown in Figs. 13 and 14 illustrate well that optimizing the torque amplitude is not trivial when experimentally relevant parameters are taken into account: Magnetic interactions, temperature, anisotropy, and finite diameters all have a strong influence on torque properties. We have not yet found a simple way (analytical equation, for instance) to predict what are the optimized particles when all these parameters are taken into account. Equation (20) is so far the best we could achieve, but it is not valid in the presence of magnetic interactions. When the latter are non-negligible, kinetic Monte Carlo simulations are so far the only possible way to predict what are the parameters permitted to maximize the torque amplitude.

\section{SUMMARY AND CONCLUSION}

Our paper, based on kinetic Monte Carlo simulations, has permitted the enlightenment of several properties of interest on the torque undergone by assemblies of MNPs. Basic properties of isolated ferromagnetic MNPs were first recalled. In this case, as illustrated in Figs. 2 and 3 of the original paper, maximizing the torque only requires maximizing the MNP anisotropy and the magnetic field. The torque value then equals the smaller between $K_{\text {eff }} V$ and $\tau_{\text {max }}$, which depends on whether $\mu_{0} H_{\max }$ is greater or smaller than $\frac{K_{\text {eff }}}{M_{S}}$. Although this is strictly true for an easy axis on the plane of the field only, the fact that a single MNP is randomly oriented does not change drastically this result (see Fig. 4 of the original paper). When thermal activation enters into play, its effects are to induce a 

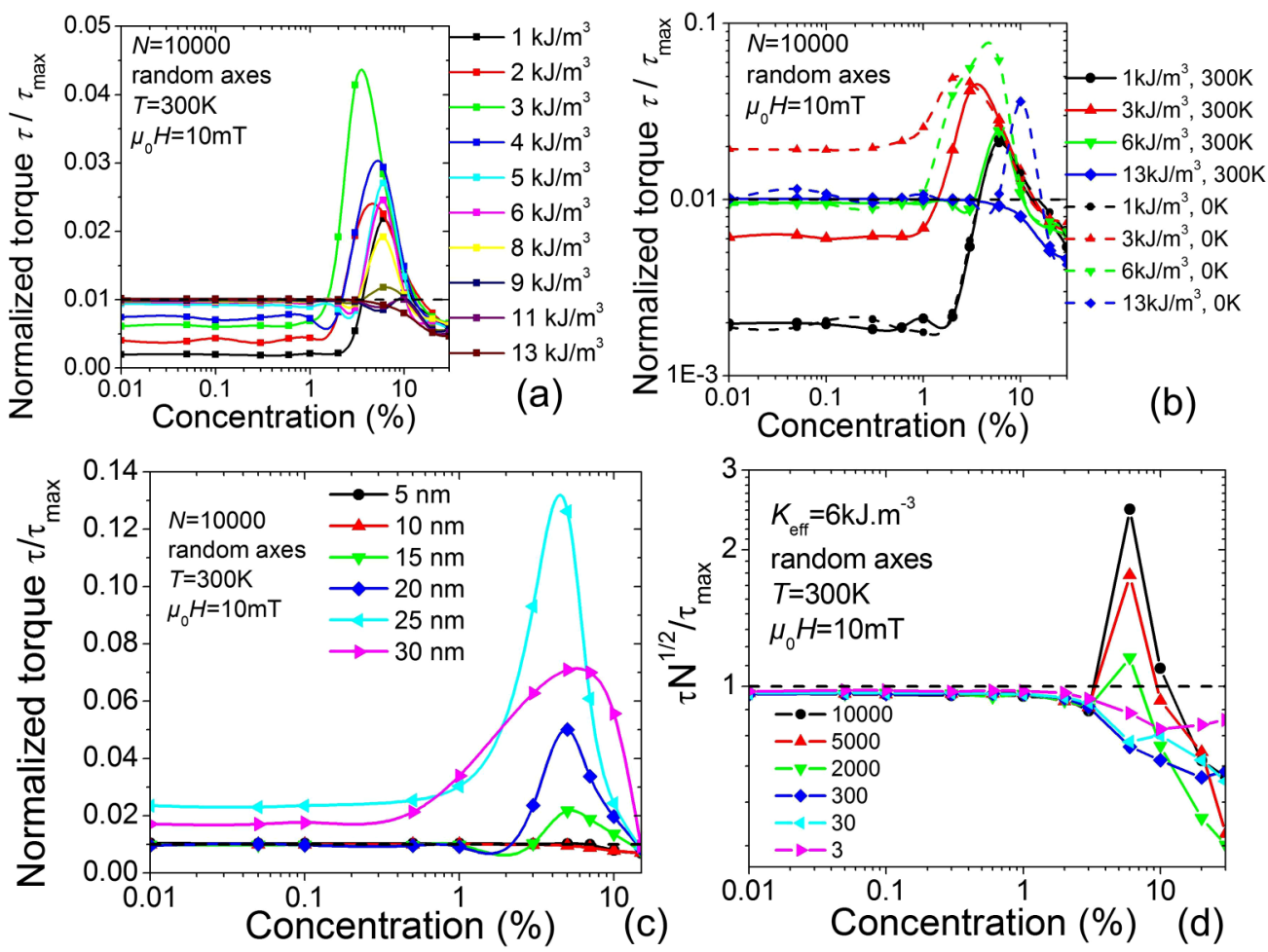

FIG. 14. Influence of the concentration on the torque undergone by an assembly of $N=10000$ interacting superparamagnetic particles with randomly oriented axes at $T=300 \mathrm{~K}$. Unless otherwise specified in the legend, $d=10 \mathrm{~nm}, \mu_{0} H_{\max }=10 \mathrm{mT}$, and $M_{S}=0.5 \times 10^{6} \mathrm{~A} / \mathrm{m}$. (a) Normalized torque for $K_{\text {eff }}$ varying between 1 and $13 \mathrm{~kJ} / \mathrm{m}^{3}$. (b) Comparison of the normalized torque at $T=300 \mathrm{~K}$ and the one at $T=0 \mathrm{~K}$ for $K_{\text {eff }}$ of $1,3,6$, and $13 \mathrm{~kJ} / \mathrm{m}^{3}$. (c) Normalized torque for a NP diameter varying between 5 and $30 \mathrm{~nm}$ for $K_{\text {eff }}=13 \mathrm{~kJ} / \mathrm{m}^{3}$. (a)-(c) The horizontal dashed lines represent the function $\frac{|\tau|}{\tau_{\max }}=\frac{1}{\sqrt{N}}$. (d) $\frac{|\tau| \sqrt{N}}{\tau_{\max }}$ is plotted as a function of concentration for $N$ varying between 3 and 10000 for $K_{\text {eff }}=6 \mathrm{~kJ} / \mathrm{m}^{3}$. The horizontal dashed lines represent the function $\frac{|\tau| \sqrt{N}}{\tau_{\max }}=1$.

high-frequency switch of the magnetization, so the torque also changes sign at high frequencies (see Fig. 6 of our original paper), leading to a decrease in the average torque felt by the MNP. However, it might be interesting not to forget that the instantaneous torque undergone by superparamagnetic NPs is the same as the one of otherwise equivalent ferromagnetic NPs (see Fig. 6 of our paper). An equation that permits calculating this average torque as well as its domain of validity has been proposed and compared to simulations [see Figs. 7, 8, and Eq. (19) of our paper].

The case of an assembly of MNPs is more complex. First one can note that, in the angle dependence of the torque, there appears a reminiscence of single MNP properties: The $\sin ^{2}(2 \phi)$ or the $\sin ^{2}(\phi)$ signature of the torque in single MNPs is still visible in the angle dependence of the torque of an assembly of 10000 MNPs [see Fig. 9(a) of the original paper]. Interestingly, simulations clearly show that there is a way to enhance the torque in an assembly of MNPs: The time-reversal symmetry must be broken. In this case, the torque undergone by the assembly can become very large due to an increase in the $\mathrm{Z}$ component of the torque. This enhancement is possible only if the magnetic field rotates not if it oscillates (see Fig. 10 of the original paper) and occurs for ferromagnetic particles when the condition $\frac{\mu_{0} H_{\max } M_{S}}{2}<K_{\text {eff }}<\mu_{0} H_{\max } M_{S}$ is fulfilled. For ferromagnetic particles, the torque is maximized when $K_{\text {eff }} \approx \mu_{0} H_{\max } M_{S}$.
When thermally activated switches are possible, the torque is maximized when there is one or two switches during the magnetic-field rotation. An equation has been proposed which permits estimating when this occurs, and good agreement with the simulations has been found [see Fig. 12(a) and Eq. (20) of our paper].

In assemblies of $N$ MNPs, in most cases, the total torque increases as $\sqrt{N}$, and the torque per unit of volume thus decreases as $\frac{1}{\sqrt{N}}$. However, this rule suffers two noticeable exceptions: (i) If all the easy axes are aligned, the total torque scales as $N$, and the torque per unit of volume is constant. This means that, whenever it is possible experimentally, this situation should be searched. For instance, it could be useful to synthesize beads in which MNPs have aligned anisotropy axes. Also, during in vitro experiments, it might be interesting to apply a constant magnetic field during the MNP accumulation at the cell membrane or inside cells to favor the alignment of easy axes during biological experiments. (ii) When the time-reversal symmetry is broken, the total torque grows more rapidly than $\sqrt{N}$; an increase following $N^{0.78}$ was extracted from simulations in optimized conditions for noninteracting ferromagnetic NPs (see Fig. 11 of our paper).

The presence of magnetic interactions makes the problem more complex. First, the study of ferromagnetic NPs without anisotropy shows that magnetic interactions have a tendency to increase the global anisotropy but also to decrease the 
magnetization of the assembly, giving a bell-shaped dependence to the concentration dependence of the torque [see Fig. 13(a)]. The latter is maximized when $\frac{c M_{S}}{H_{\max }} \approx 4$. The fact that the interactions increase the anisotropy has a consequence for ferromagnetic MNPs with a finite anisotropy: As shown in Fig. 13(b), there is a range of concentrations where the torque amplitude is increased compared to its value for independent MNPs. For very high concentrations, whatever the anisotropy of the particles, a decrease in magnetization and thus in the torque is observed.

Finally, the presence of magnetic interactions on superparamagnetic NPs, which is the most relevant for applications, is very interesting. In almost all cases, interactions increase strongly the torque above the value of $\tau=\frac{\tau_{\max }}{\sqrt{N}}$. This means that, for a given concentration which depends on several parameters ( $d, K_{\text {eff }}, N$, and $\mu_{0} H_{\text {max }}$, see Fig. 14), they display a breaking of the time-reversal symmetry and an increase in the $Z$ component of the torque. Interestingly, it occurs even for particles with $K_{\text {eff }}>\mu_{0} H M_{S}$, which do not display any breaking of time reversal at $T=0 \mathrm{~K}$ when they are ferromagnetic. Everything happens as if large anisotropy NPs in the superparamagnetic regime in a given range of concentrations behave exactly as low anisotropy noninteracting ferromagnetic NPs. It is thus possible to enhance greatly the torque generated by superparamagnetic NPs at the condition to adjust their structural characteristics.
As a conclusion, the kinetic Monte Carlo simulations that we have developed have permitted us to get an insight into the microscopic phenomena responsible for the torque amplitude in the assembly of MNPs. The problem is much more complex than one could have thought initially, but we have successfully evidenced important trends and provided analytical formulas that permit performing calculations when simulations are not available. The concepts presented in this Erratum should help chemists and biologists in synthesizing nano-objects with optimized torque properties. For physicists, it would be interesting to test experimentally the results described in this Erratum. For this purpose, torque measurements on well-characterized assemblies of nanoparticles should be performed and compared to numerical simulations.

\section{ACKNOWLEDGMENTS}

M. Anand (Indian Institute of Technology, New Delhi) has discovered the error in the program when working on the comparison between analytical equations describing the dynamical behavior of nanoparticles in the presence of interactions and kinetic Monte Carlo simulations. He is acknowledged for finding the origin of this discrepancy. A. Hillion (LPCNO, Toulouse) is acknowledged for his work running the corrected version of the program. Both will present results obtained with the corrected version of the program in forthcoming articles.

[1] F. Luis, F. Petroff, J. M. Torres, L. M. García, J. Bartolomé, J. Carrey, and A. Vaurès, Phys. Rev. Lett. 88, 217205 (2002). 